Doug Geisler, Eva K. Grebel, and Dante Minniti, eds.

\title{
Formation of Globular Clusters in Turbulent Molecular Clouds
}

\author{
Michael P. Geyer \& Andreas Burkert \\ Max-Planck-Institut für Astronomie, Königstuhl 17, D-69117 \\ Heidelberg, Germany, geyer@mpia-hd.mpg.de
}

\begin{abstract}
The formation of massive stellar clusters in turbulent molecular clouds is investigated. We include artificial star formation and energy feedback of newly born stars. The obtained systems are not likely to survive. Case studies to determine conditions necessary for forming bound clusters will be done in the future.
\end{abstract}

\section{Introduction}

The formation of globular clusters is still an unsolved puzzle. Massive bound stellar systems can only form by a time coherent collapse of large regions of molecular clouds.

Using smoothed particle hydrodynamical simulations (SPH) we investigate the formation of stellar clusters in self-gravitating collapsing turbulent molecular clouds. We mimick star formation by creating N-body particles from gas particles in regions where the the gas density exceeds the Jeans criterion and where the gas flow is convergent.

Shortly after the formation of the first stars, the remaining gas will be expelled by energy feedback of the massive stars, like ionizing radiation, stellar winds or supernova explosions. As a result of this gas expulsion phase some or all stars in the cluster will also become unbound. The fraction of finally bound stars is mainly determined by the efficiency of star formation and the timescale of the gas expulsion.

\section{Setup, Star Formation \& Feedback}

We initially start with a homogeneous gas sphere with a superimposed turbulent velocity field, created as a Gaussian random field with power spectrum $P(k) \sim k^{-2}$, where $k$ is the wave number. The turbulence decays and high density regions build up due to self-gravitation and fragmentation. The initial parameters are given in table 1 . We use an isothermal equation of state. The model can be scaled to various initial conditions.

To follow the formation process of a star cluster from scales as large as a molecular cloud down to the stars itself we need a recipe to mimick star formation. Different approaches concerning the inclusion of star formation in SPH simulations are discussed by, e.g., Katz (1992), Bate (1995), Klessen (2000), and Nakasato, Mori \& Nomoto (2000). In our simulations, whenever the density 
Table 1. Initial parameters

\begin{tabular}{ll}
\hline gas mass & $M=0.510^{5} M_{\odot}$ \\
radius & $R=50 \mathrm{pc}$ \\
time unit & $\hat{t}=1.710^{7} \mathrm{yrs}$ \\
temperature & $T=10 \mathrm{~K}$ \\
initial Jeans mass & $M_{j}=51 M_{\odot}$ \\
SPH particle number & $n=26200$ \\
\hline
\end{tabular}

of a gas particle exceeds the Jeans limit and the flow of the nearest neighbours is convergent, a collisionless $\mathrm{N}$-body particle is created with a mass according to a given intrinsic star formation efficiency. The masses of the gas particle and of its neighbours are reduced accordingly.

After a massive star has formed, it will feed back energy into the interstellar medium by ionizing radiation, stellar winds or supernova explosions. A survey of different methods implementing feedback in SPH is given by Thacker \& Couchman (2000). In our simulations each $\mathrm{N}$-body particle contributes energy to its surrounding by increasing the thermal energy of the neighbouring gas particles at a constant rate.

\section{Dynamical Evolution}

Fig. 1 shows a time sequence of one simulation. The turbulence decays and the cloud starts collapsing. At $t=1.36$ the first stars form in high density regions. Their energy feedback expells the surrounding gas and causes star formation in the compressed medium in front of outwards propagating gas shells. The newly born stars gain high velocities and are not bound in the system of stars and gas.

Fig. 2 compares the star formation efficiency with and without energy feedback. In accordance with observations, our model including energy feedback shows only low star formation efficiencies. Thus, the star cluster that has formed is not likely to survive (Lada, Margulis \& Dearborn 1984, Geyer \& Burkert 2001). This result is independent of the detailed assumption of feedback or the initial condition. It demonstrates that the formation of massive bound clusters requires conditions that are not typically found in molecular clouds. In future simulations we will investigate the requirements necessary for forming massive bound clusters.

\section{References}

Bate, M.R. 1995, PhD Thesis, Clare College, Cambridge.

Geyer, M.P. \& Burkert, A. 2001, MNRAS, 323, 988

Katz, N. 1992, MNRAS, 391, 502

Klessen, R.S. \& Burkert, A. 2000, ApJS, 128, 287

Lada, C. J., Margulis, M., Dearborn, D. 1984, ApJ, 285, 141

Nakasato, N., Mori, M. \& Nomoto, K. 2000, ApJ, 535, 776

Thacker, R.J., Couchman, H.M.P. 2000, ApJ, 545, 728 

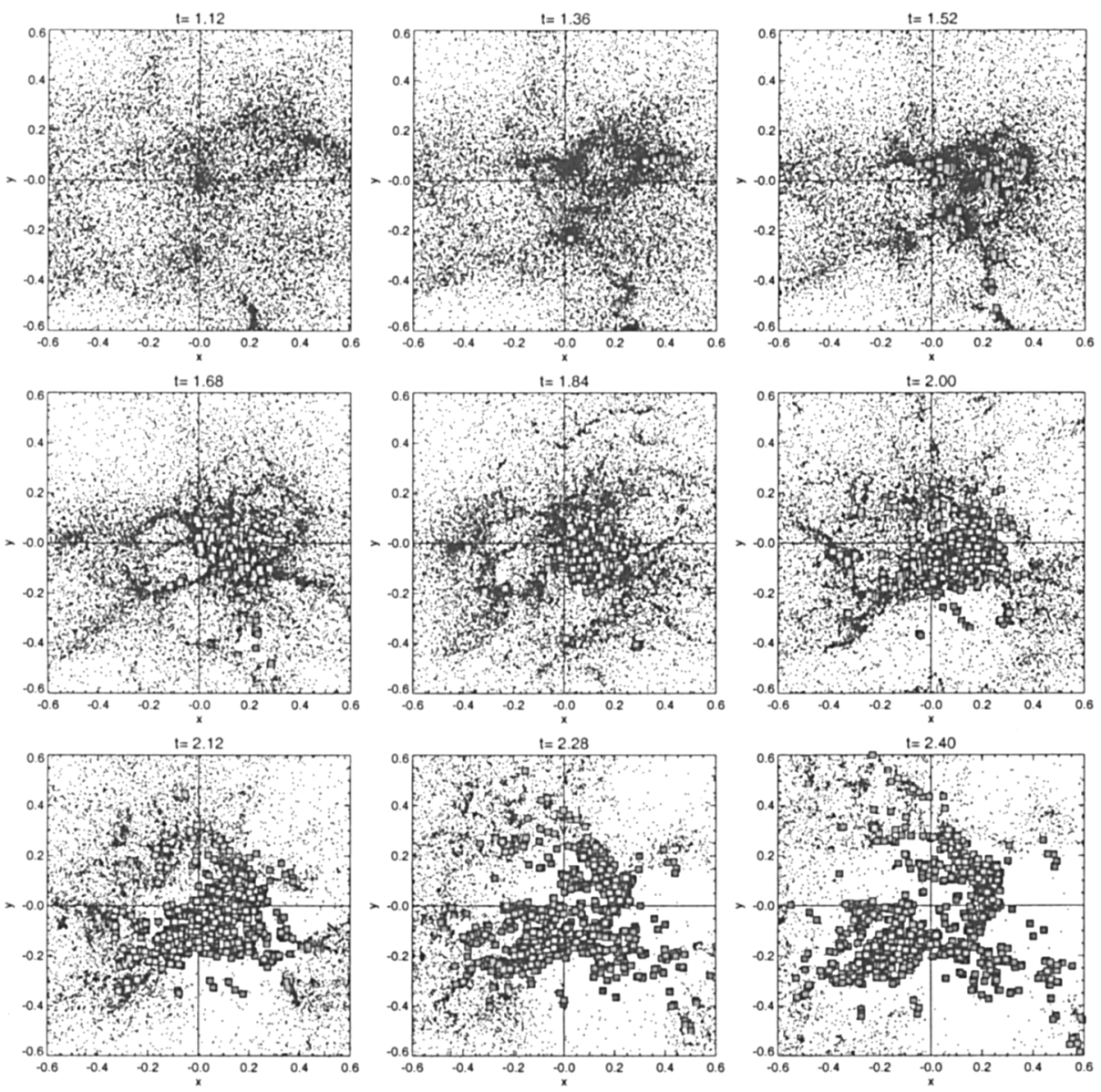

Figure 1. Time evolution projected onto the $x-y$-plane. Dots and gray squares represent gas and $\mathrm{N}$-body particles, respectively.

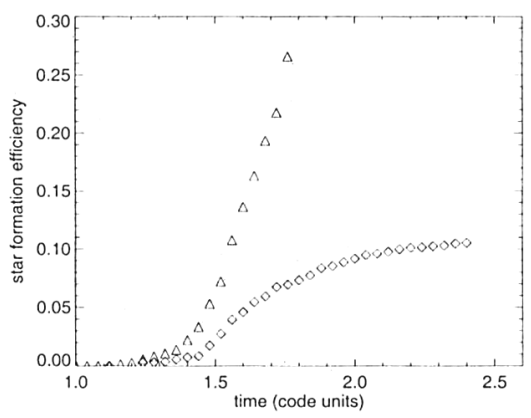

Figure 2. Time evolution of the star formation efficiency (ratio of the mass in stars to initial gas cloud mass) without (triangles) and with (diamonds) energy feedback. 